(C)2009 IEEE. Personal use of this material is permitted. However, permission to reprint/republish this material for advertising or promotional purposes or for creating new collective works for resale or redistribution to servers or lists, or to reuse any copyrighted component of this work in other works must be obtained from the IEEE. 


\section{Adaptive RLMS Algorithm for Antenna Array Beamforming}

\author{
Jalal Abdulsayed Srar \\ Dep. of Electrical and Computer Engineering \\ Curtin University of Technology \\ Perth, WA City \\ jalal.srar@postgrad.curtin.edu.au \\ jalal.srar@google.com
}

\author{
Kah-Seng Chung \\ Dep. of Electrical and Computer Engineering \\ Curtin University of Technology \\ Perth, WA City \\ k.chung@curtin.edu.au
}

\begin{abstract}
This paper presents a flexible method of achieving either fixed or self-adaptive antenna beamforming. It involves the use of an array image factor $A_{d}^{\prime}$, which interfaces an RLS and LMS sections in cascade to form the RLMS beamforming algorithm. It is shown that an accurate fixed beam can be obtained by prior setting the elements of $A_{d}^{\prime}$ with prescribed values for the required direction. Moreover, the beam direction can also be made adaptive to automatically track the target signal. In this case, a convenient and effective method is described for computing the element values of $A_{d}^{\prime}$ based on the estimated RLS output signal and tap weights. Analytical and computer simulation results verify these two modes of operation of the RLMS beamforming algorithm. Furthermore, the convergence of RLMS is shown to be quite insensitive to variations in SNR of the input signal as well as the step sizes associated with the RLS and LMS sections.
\end{abstract}

Keywords-component; Adaptive beamforming; smart antenna; LMS; RLS; RLMS algorithms.

\section{INTRODUCTION}

Modern wireless communication systems are becoming progressively more complex, in an attempt to meet growing demand for higher data rates, wider coverage and greater capacity. Recently, adaptive or smart antennas have been introduced to exploit the spatial domain for minimizing interferences thereby enhancing system coverage and capacity. These antennas automatically direct their beam patterns to the desired signals with nulls in the directions of interfering signals. The ability of these antennas to track their target signals quickly and accurately depends largely on the performance of the beamforming algorithm employed. Furthermore, the use of electronically steerable antenna arrays lessens the burden of skill shortage for installing fixed wireless communication backhaul in remote rural areas.

A steerable antenna array which uses the simultaneous perturbation stochastic approximation algorithm to tune electronically the reactances of individual parasitic elements is described in [1]. In [2], dual orthogonal polarization diversity antenna elements are used in the front-end of a discrete cylindrical lens array to produce a fan shaped fixed beam.
Various adaptive algorithms, including LMS and RLS, have also been introduced for adaptive beamforming [3]. Recently, variants of LMS and RLS have been investigated to enhance the convergence and tracking ability in time varying environments. This includes the use of a variable step size LMS algorithm (VSSLMS) in the presence of nonstationary noise [4]; a constrained constant modulus RLS algorithm for blind adaptive beamforming [4], and a hybrid scheme involving sample matrix inversion for initializing the LMS algorithm for fast convergence [5].

In [6], the authors proposed and analyzed a novel configuration, called RLMS, by cascading RLS and LMS algorithms through an array image factor $\boldsymbol{A}_{\boldsymbol{d}}^{\prime}$, as shown in Fig. 1. Computer simulations presented in $[6,7]$ show that the new RLMS algorithm converges faster than either the RLS or LMS algorithm operating on its own.

This paper examines two different ways of using the array image factor $\boldsymbol{A}_{d}^{\prime}$ to provide a flexible mean of achieving either fixed or self-adaptive antenna beamforming. In one case, the elements of $\boldsymbol{A}_{d}^{\prime}$ are prescribed with values that correspond to the desired direction. For clarity, this way of operation of RLMS is referred to in this paper as $\mathrm{RLMS}_{1}$.

For the case of a moving target, it is necessary that $\boldsymbol{A}_{d}^{\prime}$ is made adaptive in order to follow the angle of arrival (AoA) of the desired signal. A simple yet effective method of estimating $\boldsymbol{A}_{d}^{\prime}$ is presented in this paper. To differentiate from RLMS ${ }_{1}$, this new approach will from here on be simply referred to as RLMS.

The rest of the paper is organized as follows. In section II, the use of the prescribed element values of $\boldsymbol{A}_{d}^{\prime}$ for fixed beamforming is analyzed. Section III describes a simple method for deriving $\boldsymbol{A}_{d}^{\prime}$ from the estimated RLS output signal $y_{R L S}$. An analysis on the convergence of RLMS is presented in section IV. Computer simulation results, obtained for an eight-element linear array, are presented in Section $\mathrm{V}$ for both fixed and self-adaptive beamforming. Finally, Section VI concludes the paper. 


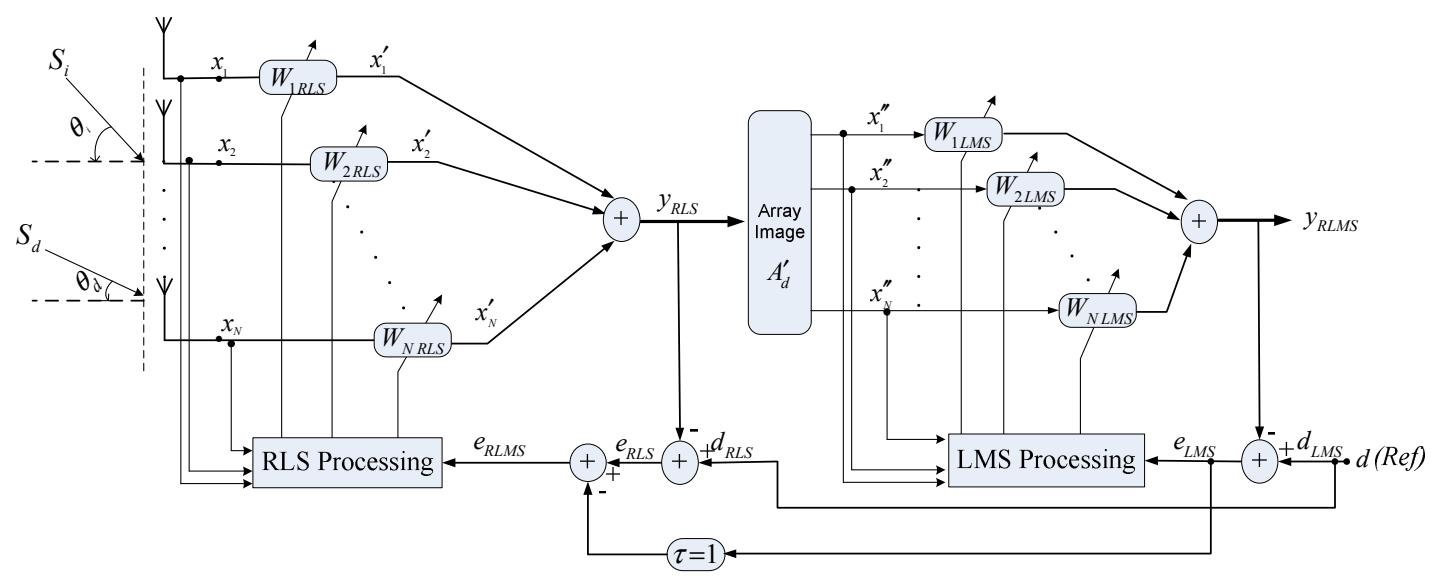

Figure 1. A configuration of the RLMS algorithm

\section{FIXED BEAMFORMING USING THE RLMS ALGORITHM}

Let the desired signal $s_{d}(t)$ and a cochannel interference $s_{i}(t)$, both originated from a distance, to impinge on an Nisotropic element linear array at an angle $\theta_{d}$ and $\theta_{i}$, respectively, as shown in Fig. 1. The resulting outputs of the individual antenna elements in the presence of AWGN, $\mathbf{n}(t)$ of variance $\sigma^{2}$ can be expressed as [8]

$$
\begin{aligned}
\mathbf{x}(t) & =\left[x_{1}(t), x_{2}(t), \ldots, x_{N}(t)\right]^{H} \\
& =\mathbf{A}_{d} s_{d}(t)+\mathbf{A}_{i} s_{i}(t)+\mathbf{n}(t)
\end{aligned}
$$

where $\boldsymbol{A}_{d}$ and $\boldsymbol{A}_{i}$ are the array factors for the desired signal and the cochannel interference, respectively. $(\cdot)^{H}$ denotes the complex conjugate transpose.

With reference to the first antenna element, $A_{d}$ and $\boldsymbol{A}_{i}$ are given by

$$
\begin{aligned}
& \boldsymbol{A}_{d}=\left[1, e^{-j \psi_{d}}, e^{-2 j \psi_{d}}, \ldots, e^{-(N-1) j \psi_{d}}\right]^{H} \\
& \boldsymbol{A}_{i}=\left[1, e^{-j \psi_{I}}, e^{-2 j \psi_{I}}, \ldots, e^{-(N-1) j \psi_{I}}\right]^{H}
\end{aligned}
$$

where $\psi_{d}=2 \pi\left(d \sin \left(\theta_{d}\right) / \lambda\right)$, and $\psi_{i}=2 \pi\left(d \sin \left(\theta_{i}\right) / \lambda\right)$. The antenna element spacing is $d$, and $\lambda$ is the carrier wavelength.

According to Fig. 1, the input stage of the RLMS scheme is based on the RLS algorithm with its weight vector $\left(\boldsymbol{W}_{R L S}\right)$ at the $(j+1)^{t h}$ iteration updated according to [9]

$$
\boldsymbol{W}_{R L S}(j+1)=\boldsymbol{W}_{R L S}(j)+\boldsymbol{p}(j+1) \boldsymbol{X}(j) e_{R L S}^{*}(j)
$$

where $\boldsymbol{X}(j)$ is the input signal vector, and $\boldsymbol{p}(j+1)$ is an arbitrary symmetric positive definite matrix given by

$$
\boldsymbol{p}(j+1)=\frac{1}{\alpha}\left[\boldsymbol{p}(j)-\frac{\boldsymbol{p}(j) \boldsymbol{X}(j) \boldsymbol{X}^{H}(j) \boldsymbol{p}(j)}{\alpha+\boldsymbol{X}^{H}(j) \boldsymbol{p}(j) \boldsymbol{X}(j)}\right]
$$

$\boldsymbol{p}(j)$ is initialized as $\delta^{-1} \boldsymbol{I}$, with $\delta$ being a small positive constant, $\alpha$ is the forgetting factor, and $\boldsymbol{I}$ is an $N \times N$ identity matrix. In this paper, $\alpha$ is assumed unity.

Now, the RLS output at the $j^{\text {th }}$ iteration is given by

$$
y_{R L S}(j)=\boldsymbol{W}_{R L S}^{H}(j) \boldsymbol{X}(j)
$$

From this estimated RLS output signal, the input signal vector for the subsequent LMS section can be obtained, such that

$$
\boldsymbol{X}_{L M S}=\boldsymbol{A}_{\boldsymbol{d}}^{\prime} y_{R L S}
$$

The LMS weight vector is updated according to

$$
\boldsymbol{W}_{L M S}(j+1)=\boldsymbol{W}_{L M S}(j)+\mu \boldsymbol{X}_{L M S}(j) e_{L M S}(j), 0<\mu<\mu_{0}
$$

where $\mu_{0}$ is a positive constant with its value dependent on the input signal statistics.

Finally, the output of the RLMS beamformer becomes

$$
\begin{aligned}
y_{R L M S} & =\boldsymbol{W}_{L M S}^{H} \boldsymbol{X}_{L M S} \\
& =\boldsymbol{W}_{L M S}^{H} y_{R L S} \boldsymbol{A}_{\boldsymbol{d}}^{\prime}
\end{aligned}
$$

Equation (9) indicates the central role played by the image array factor $\boldsymbol{A}_{d}^{\prime}$ in beamforming using the RLMS algorithm. Now, by prescribing the individual elements of $\boldsymbol{A}_{\boldsymbol{d}}^{\prime}$ with values corresponding to the required AoA, the resulting output will contain only those signal components "selected" by $\boldsymbol{A}_{d}^{\prime}$. For example, by setting $\boldsymbol{A}_{d}^{\prime}=\boldsymbol{A}_{\boldsymbol{d}}$, a fixed beam pointing in the direction of $\theta_{d}$ is thus obtained. With this scheme, variations in operating condition and component tolerance are compensated for through adaptive adjustments of the RLS and LMS tap weights.

\section{Estimation of ARray Image Factor $\boldsymbol{A}_{\boldsymbol{d}}^{\prime}$}

For self-adaptive beamforming, it requires that $A_{d}^{\prime}$ be adjusted automatically to always track the AoA of the desired signal. A simple method for estimating $\boldsymbol{A}_{\boldsymbol{d}}^{\prime}$ is now described. 
In the following derivation, the tap weights are assumed randomly initialized.

Now, rearranging (1) in element form gives

$$
x_{k}(t)=A_{d, k} s_{d}(t)+A_{i, k} s_{i}(t)+n(t)
$$

where $A_{d, k}$ is the $k^{t h}$ element of $\boldsymbol{A}_{d}$ with $k=1,2, \ldots, N$.

The outputs of the individual RLS taps $\left(w_{i}\right)$ are given by

$$
x_{k}^{\prime}(t)=w_{k} x_{k}(t)
$$

When RLS converges, the output $y_{R L S}$ tends to approach $s_{d}(t)$ with both the interference $s_{i}(t)$ and noise $\mathrm{n}(\mathrm{t})$ being suppressed. Thus, let $y_{R L S}(t) \simeq s_{d}(t)$, and using the estimation on both sides of (10) yields

$$
E\left[x_{k}(t)\right]=A_{d, k} E\left[s_{d}(t)\right] \simeq A_{d, k} y_{R L S}(t)
$$

where $E[\cdot]$ denotes expectation.

By assuming that both the input signal $(\boldsymbol{X})$ and the RLS weights $(\boldsymbol{W})$ are independent, the expectation of (11) can be written as

$$
E\left[x_{k}^{\prime}(t)\right]=E\left[w_{k}\right] E\left[x_{k}(t)\right]
$$

From (12) and (13), the array factor elements are estimated as

$$
A_{d, k}(t) \simeq \frac{E\left[x_{k}^{\prime}(t)\right]}{E\left[w_{k}\right] y_{R L S}(t)}
$$

It follows that the instantaneous values of the elements of $\boldsymbol{A}_{d}$ can be expressed as

$$
A_{d, k}(t) \simeq \frac{x_{k}^{\prime}(t)}{w_{k}(t) y_{R L S}(t)}
$$

Thus, (15) provides a mean of calculating the array image factor $\boldsymbol{A}_{d}^{\prime}$ for use in the RLMS algorithm.

\section{CONVERGENCE OF THE RLMS ALGORITHM}

In [6], it is shown that the mean-square error $\xi$ converges to the minimum MSE, $\xi_{\min } . \xi$ is defined here as the expected value of $e_{R L M S}^{2}$, such that

$$
\begin{aligned}
\xi= & \sum_{i=1}^{j} \alpha^{j-i}\left\{E\left[D^{2}(i)\right]-2 E\left[D(i) \boldsymbol{X}^{H}(i) \boldsymbol{W}_{R L S}(j)\right]\right\} \\
& +\boldsymbol{W}_{R L S}^{H}(j) \boldsymbol{Q}(j) \boldsymbol{W}_{R L S}(j)
\end{aligned}
$$

and,

$$
\begin{aligned}
\xi_{\min }= & \sum_{i=1}^{j} \alpha^{j-i}\left\{\left|d_{R L S}(i)\right|^{2}+\left|d_{L M S}(i-1)\right|^{2}\right\} \\
& +\boldsymbol{W}_{R L M S}^{H}(j-1) \boldsymbol{Z}(j-1)\left\{-2+\boldsymbol{A}_{d}^{\prime H} \boldsymbol{W}_{L M S}(j-1)\right\} \\
& -\boldsymbol{Z}^{H}(j) \boldsymbol{W}_{\text {opt }_{R L S}}(j)
\end{aligned}
$$

where $\boldsymbol{W}_{L M S}^{H}$ and $\boldsymbol{W}_{R L S}^{H}$ are the complex conjugate transpose of the weight vectors for the LMS and RLS algorithms, respectively, and

$$
\begin{gathered}
D(j)=d_{R L S}(j)-e_{L M S}(j-1) \\
e_{R L S}(j)=d_{R L S}(j)-\boldsymbol{W}_{R L S}^{H}(j) \boldsymbol{X}(j) \\
e_{L M S}(j)=d_{L M S}(j)-\boldsymbol{W}_{L M S}^{H}(j) \boldsymbol{X}_{L M S}(j) \\
\boldsymbol{W}_{R L M S}^{H}=\boldsymbol{W}_{L M S}^{H} \boldsymbol{A}_{d} \boldsymbol{W}_{R L S}^{H}
\end{gathered}
$$

Also, the correlation matrix of the input signals is given by

$$
\boldsymbol{Q}(j)=\sum_{i=1}^{j} \alpha^{j-i} \boldsymbol{X}(j) \boldsymbol{X}^{H}(j)
$$

The input signal cross-correlation vector is

$$
\boldsymbol{Z}(j)=\sum_{i=1}^{j} \alpha^{j-i} \boldsymbol{X}(j) d^{*}(j)
$$

where $d(j)$ and $d^{*}(j)$ are the reference signal and its conjugate, respectively.

Now, let $\boldsymbol{A}_{d}^{\prime}=\boldsymbol{\beta} \boldsymbol{A}_{d}$, where $\beta$ is a complex number which indicates the ratio of the estimated output $y_{R L S}$ and the desired signal $s_{d}(t)$. For example, when $y_{R L S}=s_{d}(t), \beta=1$, i.e., the estimated array factor $\boldsymbol{A}_{d}^{\prime}$ is the same as the actual array factor $\boldsymbol{A}_{\boldsymbol{d}}$. Replacing $\boldsymbol{A}_{\boldsymbol{d}}$ with the estimated $\boldsymbol{A}_{d}^{\prime}$ and following the same analysis as described in [6], it can be shown that the mean square error $\xi$, as specified by (16), can be expressed as

$$
\begin{aligned}
\xi= & \sum_{i=1}^{j} \alpha^{j-i}\left\{\left|d_{R L S}(i)\right|^{2}+\left|d_{L M S}(i-1)\right|^{2}\right\} \\
& +|\beta|^{2} \boldsymbol{W}_{R L M S}^{H}(j-1) \boldsymbol{Q}(j-1) \boldsymbol{W}_{R L M S}(j-1) \\
& -2 \boldsymbol{Z}^{H}(j) \boldsymbol{W}_{R L S}(j)+\boldsymbol{W}_{R L S}^{H}(j) \boldsymbol{Q}(j) \boldsymbol{W}_{R L S}(j) \\
& -2 \beta \boldsymbol{W}_{R L M S}^{H}(j-1) \boldsymbol{Z}(j-1)
\end{aligned}
$$

By differentiating (22) with respect to the weight vector $\boldsymbol{W}_{R L S}^{H}(j)$ and equating the result to zero, we obtain the optimal vector weight $\boldsymbol{W}_{\text {opt }_{R L S}}(j)$ given by

$$
\boldsymbol{W}_{o p t_{R L S}}(j)=\boldsymbol{Q}^{-1}(j) \boldsymbol{Z}(j)
$$

This represents the Wiener-Hopf equation in matrix form. Therefore, the minimum MSE, $\xi_{\min }$ can be obtained from (23) and (22), such that

$$
\begin{aligned}
\xi_{\min }= & \sum_{i=1}^{j} \alpha^{j-i}\left\{\left|d_{R L S}(i)\right|^{2}+\left|d_{L M S}(i-1)\right|^{2}\right\} \\
& +\beta \boldsymbol{W}_{R L M S}^{H}(j-1) \boldsymbol{Z}(j-1)\left\{\beta^{*} \boldsymbol{A}_{d}^{H} \boldsymbol{W}_{L M S}(j-1)-2\right\} \\
& -\boldsymbol{Z}^{H}(j) \boldsymbol{W}_{\text {opt }_{R L S}}(j)
\end{aligned}
$$




\section{Simulation RESUlts}

The performance of RLMS and its ability to realize either fixed or self-adaptive beamforming has been investigated by means of MATLAB simulations. For comparison purposes, results have also been obtained for RLMS 1 (i.e., RLMS with fixed $\boldsymbol{A}_{d}^{\prime}$ ), LMS and RLS algorithms. For the simulations, the following parameters are used:

- A linear array of 8 isotropic antenna elements spaced half a carrier wavelength apart.

- $\quad$ The desired BPSK signal arrives at $\theta_{d}=0^{\circ}$.

- The channel is AWGN of zero mean and variance $\sigma^{2}$.

- All weight vectors are initially set to zero.

- Each simulation run involves 100 iterations.

\section{A. RLMS performance}

1) Performance with external reference: The convergence performances of the RLMS, RLMS 1 , RLS and LMS schemes achieved through the use of an external reference signal have been studied based on the ensemble average square error $\left(\tilde{e}^{2}\right)$ obtained from 100 individual simulation runs. Simulations have been carried out for three different values of input $S N R$ (i.e., 5, 10 and $15 d B$ ) with different combinations of the RLS step size, $\delta$, and LMS step size, $\mu$.

The effects of input SNR on the convergence of RLS ( $\delta=0.05$ ) and RLMS and RLMS $1(\delta=0.05, \mu=0.075$ ) algorithms are shown in Fig. 2. It is observed that all the three algorithms achieved similar convergence speed but RLS has higher error floors particularly at lower SNR values. Now, for $S N R \geq 10 \mathrm{~dB}, \mathrm{RLMS}_{1}$ and RLMS converge slightly faster than RLS with all the three schemes converge within 10 iterations. Moreover, both the RLMS 1 and RLMS have almost identical convergence performance for all the three SNR values considered. This validates the method of estimating $\boldsymbol{A}_{\boldsymbol{d}}^{\prime}$ for RLMS presented in section III.

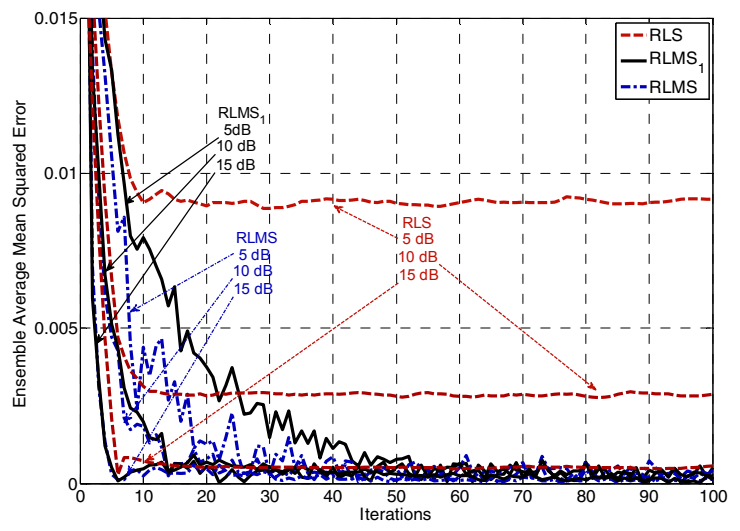

Figure 2. The convergence of RLS, RLMS 1 and RLMS with $\delta=0.05$ and $\mu=0.075$ for three different values of input $S N R$.
Furthermore, simulations have also been carried out using different combinations of $\delta(0.05,0.5$, and 1$)$ and $\mu(0.001$, 0.01 and 0.1 ). The results obtained (not shown here) verify that the operations of RLMS and RLMS 1 are quite insensitive to the choice of the step sizes used.

2) Performance with self-referencing: As shown in Fig. 2, the RLS section within the RLMS scheme converges rapidly in less than ten iterations to produce an output $y_{R L S}$, which closely resembles the input desired signal $s_{d}(t)$. This output is then used as the reference signal for the next iteration of the LMS section in $\mathrm{RLMS}_{1}$ and RLMS. As the LMS section converges, its output $y_{R L M S}$ becomes the estimated $s_{d}(t)$. This suggests that $y_{\text {RLMS }}$ may be used as the reference for the RLS section. This feedforward and feedback arrangement enables the provision of self-referencing in $\mathrm{RLMS}_{1}$ and RLMS, and allows the external referencing to be discontinued after an initial few iterations. The ability of the RLMS 1 and RLMS algorithms to maintain operation with the internally generated reference signals is demonstrated in Fig. 3, whereas both the LMS and RLS algorithms operating on their own failed to converge without the use of a correct reference signal.

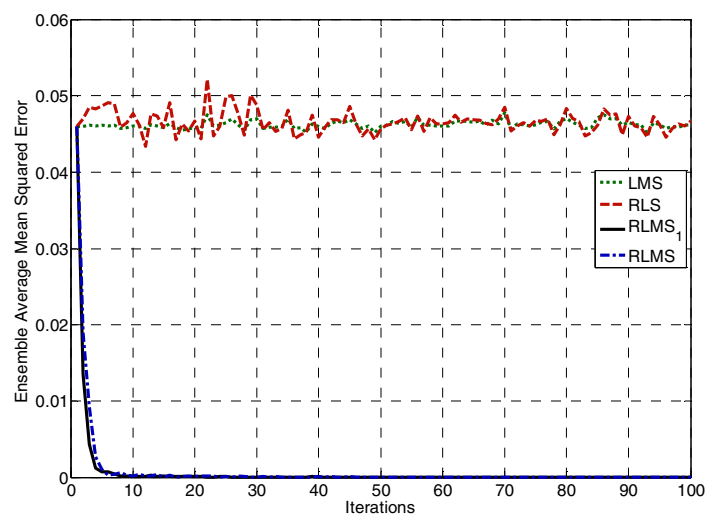

Figure 3. The convergence of RLMS and RLMS ${ }_{1}$ with self-referencing for the case of $\mu=0.075, \delta=0.05$ and $S N R=10 \mathrm{~dB}$. For comparison, no external reference is used for the two separate tests involving the LMS and RLS algorithms.

3) Performance in the presence of multiple interfering signals: Consider the desired signal that arrives at $\theta_{d}=0$ is corrupted by cochannel interference and AWGN. In this study, the rms noise level $\sigma$ is set at 0.05 giving rise to an SNR of $10 d B$. On the other hand, the interference is made up of up to four equal-amplitude cochannel interfering signals with angles of arrival (AoA) of $20^{\circ}, 45^{\circ},-30^{\circ}$ and $-50^{\circ}$. The effects of these extraneous factors on the resulting beam patterns and output signal-to-interference plus noise ratios, $S I N R_{o}$, obtained using the RLMS 1 and RLMS algorithms are examined.

Fig. 4 shows that the beam patterns obtained with RLMS and $\mathrm{RLMS}_{1}$ are almost identical except for a gain difference of 
about $2 d B$ dB between them. Also, it is observed that these algorithms are effective in suppressing close-in interference, say $\theta_{i}=20^{\circ}$, with a rejection ratio of $22 \mathrm{~dB} \mathrm{~dB}$. Furthermore, the other three interfering signals with larger AoAs are being suppressed by a greater degree. Note that the interference suppression of RLS is similar to that of RLMS while LMS has the poorest performance.

Next, the resultant values of $\operatorname{SINR}_{o}$, computed according to [4], achieved by RLMS, RLMS ${ }_{1}$, RLS and LMS in the presence of different number of interfering signals are tabulated in Table I. As to be expected, in each case, the $\operatorname{SINR} R_{o}$ deceases as the amount of interference increases. However, RLMS and $\mathrm{RLMS}_{1}$ suffer less than $1 \mathrm{~dB}$ decrease in $\operatorname{SINR}_{o}$ when the number of interfering signals is increased from one to four. Among the four schemes, LMS achieves the smallest $\operatorname{SINR}_{o}$.

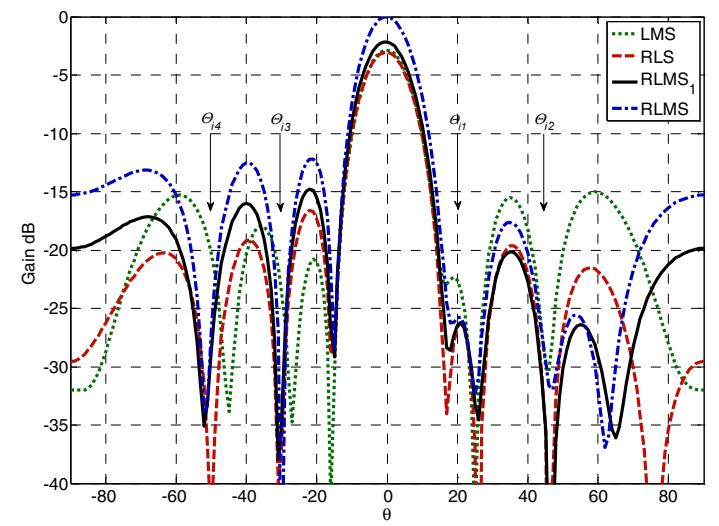

Figure 4. The beam patterns obtained with RLMS, RLMS ${ }_{1}$, RLS and LMS algorithms in the presence of four equal-amplitude interfering signals arriving

$$
\text { at } \theta_{i 1}=20^{\circ}, \theta_{i 2}=45^{\circ}, \theta_{i 3}=-30^{\circ} \text { and } \theta_{i 4}=-50^{\circ}
$$

TABLE I. SINRO (DB) ACHIEVED WITH DIFFERENT NUMBER OF INTERFERING SIGNALS

\begin{tabular}{|c|c|c|c|c|}
\hline \multirow{2}{*}{ Algorithm } & \multicolumn{4}{|c|}{ No. of Interfering Signals } \\
& 1 & 2 & 3 & 4 \\
\hline LMS & 13.46 & 11.45 & 9.70 & 8.86 \\
\hline RLS & 14.6 & 12.83 & 11.64 & 11.02 \\
\hline RLMS $_{1}$ & 15.06 & 14.63 & 14.31 & 14.27 \\
\hline RLMS & 15.51 & 15.13 & 14.94 & 14.85 \\
\hline
\end{tabular}

4) Performance with a noisy reference signal: The performances of RLMS, $\mathrm{RLMS}_{1}$, RLS and LMS have also been investigated when the reference signal used is corrupted by AWGN. This is done by examining the mean square error $\xi$ resulting from the presence of different noise level in the reference signal. Fig. 5 shows the ensemble average of the mean square error, $\bar{\xi}$, obtained from 100 individual simulation runs, as a function of the ratio of the rms noise level $\sigma$ to the amplitude of the reference signal.

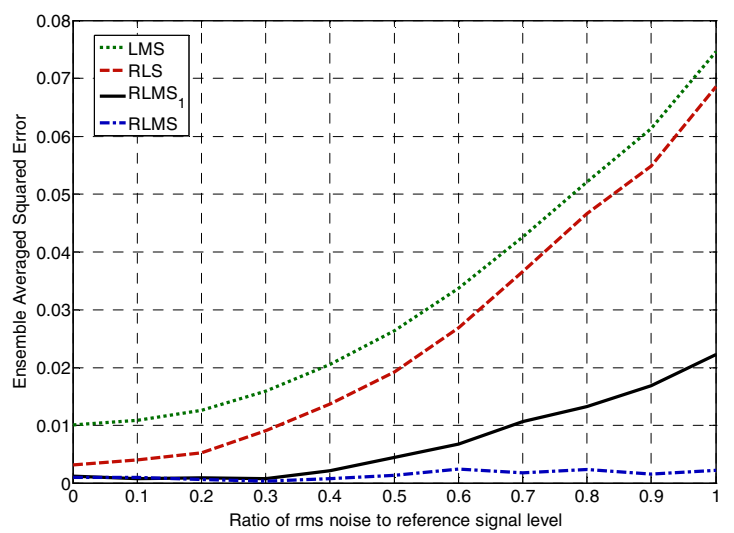

Figure 5. The influence of noise in the reference signal on the mean square error $\xi$.

It is interesting to note that both the LMS and RLS algorithms on their own are quite sensitive to the presence of noise in the reference signal. However, when they are incorporated to form the RLMS algorithm, the resulting scheme becomes very tolerant to noisy reference signal. As shown in Fig. 5, the values of $\bar{\xi}$ associated with RLMS remain very small even when the rms noise level becomes as large as the reference signal.

\section{B. Fixed beamforming}

For fixed beamforming, individual elements of $\boldsymbol{A}_{d}^{\prime}$ are being assigned values pre-calculated using (2) according to the required direction. Fig. 6 and Fig. 7 show the fixed beam patterns obtained for an input SNR of $10 d B$ when the desired direction is set to either $-20^{\circ}$ or $20^{\circ}$, and $0^{\circ}$ or $40^{\circ}$ or $60^{\circ}$, respectively. Except for the case of $\theta_{d}=60^{\circ}$, the beam patterns obtained are almost identical with a worst case side lobe gain of $-13 d B$.

Next, the beam resolution that could be achieved with the proposed scheme is investigated. The resulting beam patterns achieved for direction set at either $-2^{\circ}, 0^{\circ}, 1^{\circ}$ or $5^{\circ}$ are shown in Fig. 8. These results indicate that it is possible to differentiate very small difference in beam direction.

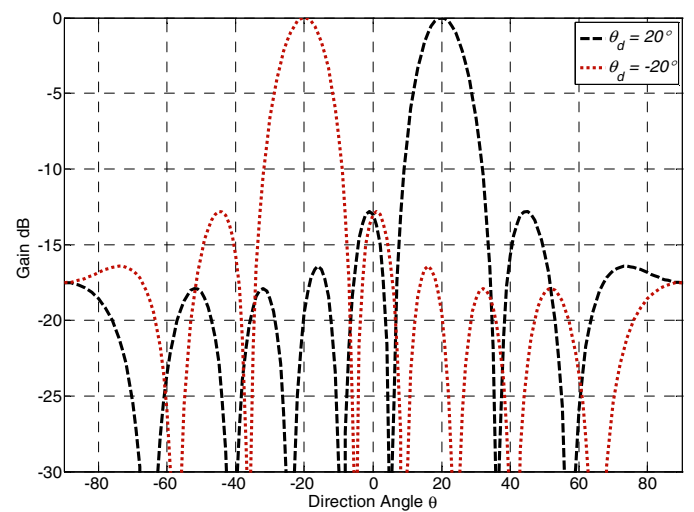

Figure 6. The beam patterns of $\mathrm{RLMS}_{1}$ obtained at $\theta_{d}=-20^{\circ}$ and $20^{\circ}$. 


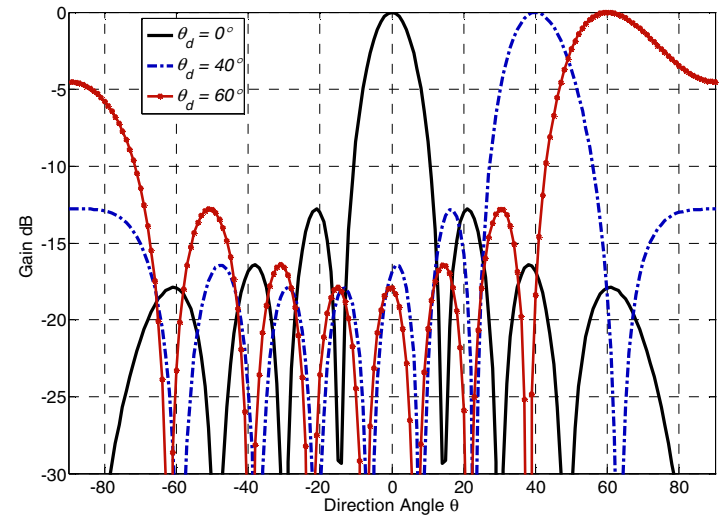

Figure 7. The beam patterns of $\mathrm{RLMS}_{1}$ obtained at $\theta_{d}=0^{\circ}, 40^{\circ}$ and $60^{\circ}$.

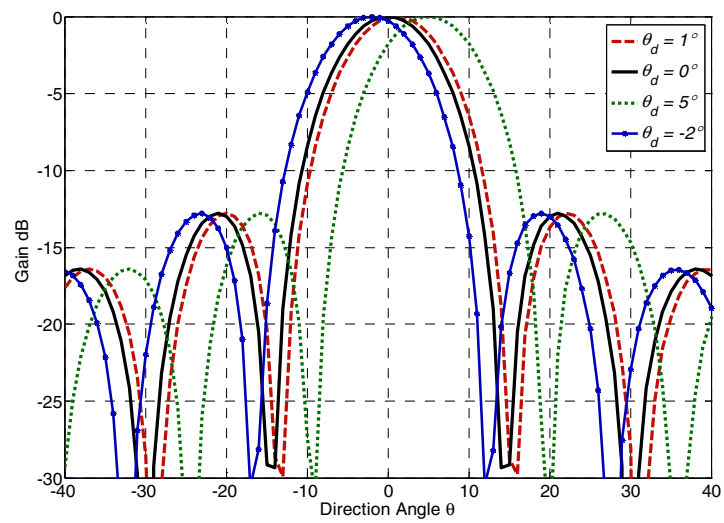

Figure 8. Beam patterns of RLMS 1 obtained at four small angles of $\theta_{d}$

$$
\left(-2^{\circ}, 0^{\circ}, 1^{\circ} \text { and } 5^{\circ}\right)
$$

\section{CONCLUSIONS}

A new and effective array beamforming algorithm, called RLMS, has been investigated analytically as well as by computer simulation. As discussed in section I, the proposed algorithm makes use of the array image factor $\boldsymbol{A}_{\boldsymbol{d}}^{\prime}$ to interface between RLS and LMS algorithms. In this way, an accurate fixed beam can be obtained by prior setting the elements of $\boldsymbol{A}_{\boldsymbol{d}}^{\prime}$ with prescribed values for the required direction. Alternatively, $\boldsymbol{A}_{\boldsymbol{d}}^{\prime}$ may be made adaptive to automatically track the target signal. A simple and effective method has been proposed for calculating the element values of $\boldsymbol{A}_{\boldsymbol{d}}^{\prime}$ based on the estimated
RLS output signal and tap weights. The convergence of RLMS has been verified analytically.

Extensive computer simulations have yielded results on the rates of convergence of RLMS (adaptive $\boldsymbol{A}_{\boldsymbol{d}}^{\prime}$ ) and RLMS (fixed $\boldsymbol{A}_{\boldsymbol{d}}^{\prime}$ ) in comparison with RLS and LMS operating individually. Simulation results indicated that both RLMS and RLMS $_{1}$ performed better than either RLS or LMS in the presence of AWGN and cochannel interference. Furthermore, both the proposed schemes are robust to noisy reference signal and may also operate with self-referencing. Unlike RLS and LMS, both RLMS and RLMS 1 are quite insensitive to the choice of the step sizes, $\delta$ and $\mu$ used.

The performance enhancements of both RLMS and RLMS have been achieved at the expense of a slight increase in computational complexity over the traditional RLS algorithm.

\section{REFERENCES}

[1] Osseiran, A. and A. Logothetis, "A Method for Designing Fixed Multibeam Antenna Arrays in WCDMA Systems", Antennas and Wireless Propagation Letters, vol. 5(1), pp. 41-44, Dec. 2006.

[2] Popovic, D. and Z. Popovic, "Multibeam antennas with polarization and angle diversity", IEEE Transactions on Antennas and Propagation", vol 50(5), pp. 651-657, 2002.

[3] Ueng, F.-B., J.-D. Chen, and S.-H. Cheng, "Smart Antenna for Multiuser DS/CDMA Communication in Multipath Fading Channels", IEICE Transactions on Communications, vol. E88, pp. 2944-2954, Jul 2005.

[4] Lei, W. and R.C. de Lamare, "Constrained Constant Modulus RLSbased Blind Adaptive Beamforming Algorithm for Smart Antennas", 4th International Symposium on Wireless Communication Systems, pp. 657661, Trondheim, Norway, Oct. 2007.

[5] Shubair, R.M., A. Merri, and W. Jessmi, "Improved adaptive beamforming using a hybrid LMS/SMI approach", Second IFIP International Conference on Wireless and Optical Communications Networks , pp. 603-606, Dubai, United Arab Emirates, Mar. 2005.

[6] Srar, J.A. and K.-S. Chung, "Adaptive Array Beam Forming Using a Combined RLS-LMS Algorithm", The 14th Asia-Pacific Conference on Communications, APCC 2008, Tokyo, Japan, Oct. 2008.

[7] Srar, J.A. and K.-S. Chung, "Performance of RLMS Algorithm in Adaptive Array Beam Forming", 11th IEEE International Conference on Communication Systems, pp. 493-498, Guangzhou, China, Nov. 2008.

[8] Litva, J. and T.K.-Y. Lo., Digital beamforming in wireless communications, Artech House, 1996.

[9] Godara, L.C., "Application of antenna arrays to mobile communications. II. Beam-forming and direction-of-arrival considerations", Proceedings of the IEEE, vol. 85(8), pp. 1195-1245, 1997. 